\title{
Regional Anaesthesia in Enhanced Recovery After Surgery Pathways - A Quintessential Component
}

\author{
Abhijit S. Nair ${ }^{1}$, Sandeep Diwan ${ }^{2}$ \\ ${ }^{1}$ Department of Anaesthesia, Ibra Hospital, Ministry of Health-Oman, Ibra, Sultanate of Oman. \\ ${ }^{2}$ Department of Anaesthesia, Sancheti Hospital, Pune, Maharashtra, India.
}

\section{Introduction:}

Enhanced recovery after surgery (ERAS) is a multimodal, perioperative care pathway designed to achieve early recovery for patients undergoing major surgery. [1] Initially described by Henry Kehlet in 1995 for colonic surgeries, the enhanced recovery pathways have now evolved and are now validated for more than 30 different types of surgery which include but are not limited to emergency laparotomy, neonatal surgeries, and lower segment cesarean sections. [2] Not only is the patient benefited from this by having an enhanced recovery and early discharge from the hospital, the cost of treatment is reduced and also leads to more turnover of patients thereby reducing the waiting list of patients scheduled for various surgeries. [3]

\section{Regional anaesthesia in ERAS pathways:}

The role of anaesthesiologists' is important in the successful implementation of preoperative, intraoperative, and postoperative pathways. [4] The success of implementing ERAS pathways lies in three factors; evidence-based perioperative care, multimodal and multi-disciplinary teamwork, and continuous audit for improving patient care. [5] The use of multimodal, opioid-sparing analgesia is emphasized in ERAS pathways using a combination of medications to reduce unwanted adverse effects like constipation, ileus, postoperative nausea/vomiting thereby facilitating early recovery and discharge. [6] Poorly managed postoperative pain is one of the important risk factors for chronic postsurgical pain (CPSP). [7,8]

As per the consensus statement for anaesthesia practice in patients undergoing gastrointestinal surgery, Feldheiser et al suggested multimodal analgesia (MMA), evidence-based and procedure-specific analgesic regimens that should be tailored with care and supported by evidence, intending to achieve optimal analgesia with minimal side effects. The goal is to achieve ERAS milestones such as early mobilization and oral feeding. [9] MMA constitutes the use of more than one analgesic modality which could be pharmacological, interventional, or adjunctive nonpharmacological techniques include acupuncture, music therapy, transcutaneous electrical nerve stimulation, and hypnosis. An important aim of MMA is the lesser use of opioids to avoid the undesirable side effects associated with its use. [10]

The two main pillars of MMA are pharmacological agents and regional anaesthesia (RA). The list of pharmacological agents is extensive. They are acetaminophen, dexamethasone, NSAIDs, IV lidocaine, $\alpha 2$ agonists like clonidine and dexmedetomidine, magnesium sulphate, nefopam, esmolol, gabapentinoids, N-methyl-d-aspartate (NMDA) receptor antagonist like ketamine. [11-13] Adding RA helps in reducing perioperative use of opioids, reduced surgical stress response, reduced postoperative nausea/vomiting (PONV), early return of bowel function due to lesser opioid use, facilitates early mobilization, reduces thromboembolic events, and leads to lesser incidence of delirium in the postoperative period. [14] [Figure 1] Table 1 depicts the various RA techniques which can be implemented for different types of surgeries.

Address of Correspondence: Dr. Abhijit S. Nair, Department of Anaesthesia, Ibra Hospital, Ministry of Health-Oman, Ibra-414, Sultanate of Oman. E-mail: abhijitnair95@gmail.com

DOI: 10.13107/ijra.2021.v02i02.033 | www.ijrajournal.com |

This is an Open Access article distributed under the terms of the Creative Commons Attribution Non-Commercial-Share Alike 4.0 License

(http://creativecommons.org/licenses/by-nc-sa/4.0) which allows others to remix, tweak, and build upon the work non-commercially as long as appropriate credit is given and the new creation are licensed under the identical terms. 


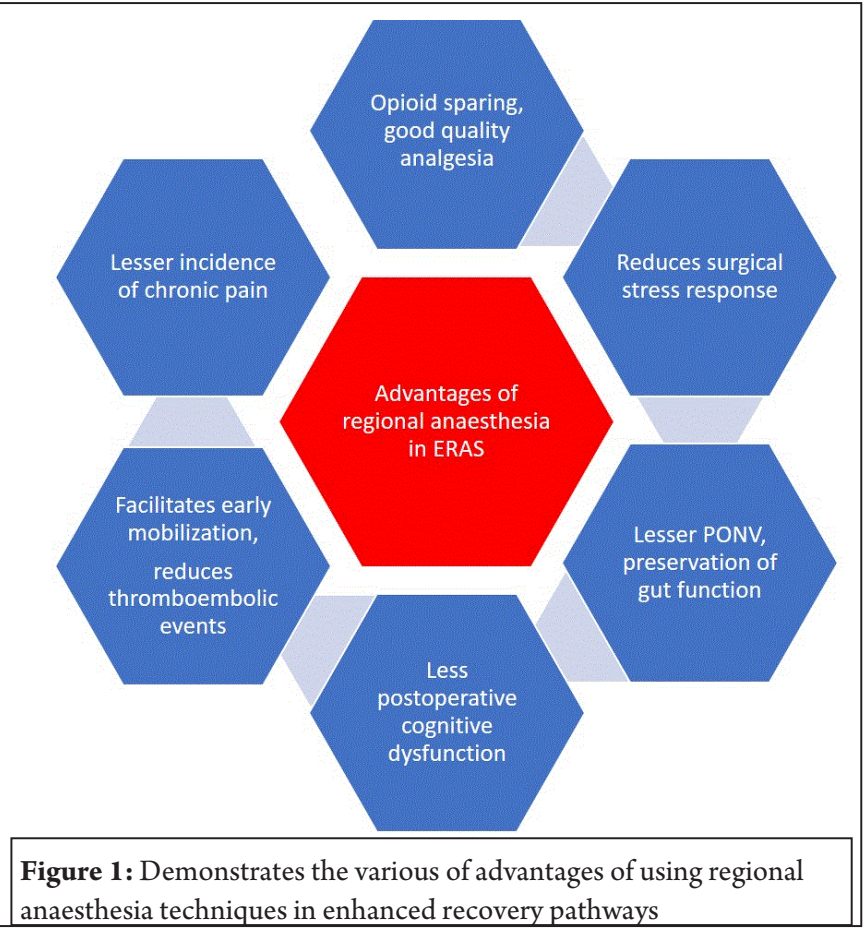

\section{Available evidence regarding implementation of RA in} ERAS:

RA includes a central neuraxial block (spinal anaesthesia, epidural anaesthesia), paraspinal and plexus blocks, peripheral nerve blocks, and the fascial plane blocks. Thoracic epidural analgesia (TEA) is considered the gold standard analgesic modality for thoracic, abdominal, and pelvic surgeries provided it is open. Although ERAS society does not recommend TEA for minimal access surgeries, it can be individualized based on the discretion of the clinician and patient profile. The advantages of TEA are lesser opioid use, early recovery of bowel function, better glycemic control, and attenuation of the surgical stress response. On the contrary, several studies which evaluated incorporating TEA as a component on MMA for laparoscopic colorectal surgeries and thoracic surgeries did not find any additional benefit of TEA. [15-17] In a dose ranging from 200-300 $\mu \mathrm{g}$, a single dose of intrathecal morphine (ITM) has been found superior to MMA including systemic opioids in patients undergoing colonic surgeries, hepato-pancreatic-biliary

\begin{tabular}{|c|c|c|}
\hline Sr. No. & Category of surgery & Various regional anaesthesia techniques \\
\hline 1 & Head and neck surgeries & $\begin{array}{l}\text { Supraorbital and supratrochlear nerve block, sphenopalatine ganglion block, infraorbital nerve block, } \\
\text { mandibular nerve block, mental nerve block, superficial cervical plexus block, glossopharyngeal nerve } \\
\text { block }\end{array}$ \\
\hline 2 & Breast surgeries & $\begin{array}{l}\text { Paravertebral block, thoracic epidural, pectoral nerve blocks (PECS } 1 \text { and 2), serratus anterior plane } \\
\text { block, erector spinae plane block, rhomboid intercostal block }\end{array}$ \\
\hline 3 & Thoracic surgeries & $\begin{array}{l}\text { Paravertebral block, thoracic epidural, serratus anterior plane block, erector spinae plane block, } \\
\text { parasternal blocks }\end{array}$ \\
\hline 4 & Abdominal surgeries & $\begin{array}{l}\text { Thoracic epidural, paravertebral block, erector spinae plane block, transversus abdominis plane block, } \\
\text { quadratus lumborum block, rectus sheath block }\end{array}$ \\
\hline 5 & Upper limb surgeries & Brachial plexus block- approach as indicated, individual nerve block as indicated \\
\hline 6 & $\begin{array}{l}\text { Lower limb surgeries } \\
\text { including arthroplasties }\end{array}$ & $\begin{array}{l}\text { Lumbar epidural, femoral nerve block, sciatic nerve block,3-in-1 block, fascia iliaca compartment block, } \\
\text { adductor canal block, iPACK block, quadratus lumborum block, lumbar erector spinae plane block, } \\
\text { lumbosacral plexus block }\end{array}$ \\
\hline 7 & Spine surgeries & Erector spinae plane block, thoracolumbar interfascial plane block \\
\hline
\end{tabular}

Table 1: depicts various regional anaesthesia techniques which can be used for various surgeries as a part of enhanced recovery pathway 
surgeries, and surgeries for gynecological malignancies. [18-

20]

The level of recommendation is high for use of lumbar epidural catheters as part of MMA in patients undergoing hip and knee arthroplasties. [21-24] Several researchers advocate the use of adductor canal block (ACB), IPACK (Infiltration between the Popliteal artery and Capsule of the Knee) block, continuous femoral nerve catheters along with MMA to facilitate early mobilization and faster recovery after arthroplasties. Although to date there is no article describing the role of RA in ERAS for arthroscopic surgeries, this area can be explored in the future to facilitate enhanced recovery after knee and shoulder arthroscopic repairs. In a meta-analysis by Edwards et al, the authors concluded that $\mathrm{ACB}$ preserves quadriceps function better than a femoral nerve block in patients undergoing ACL reconstruction with comparable analgesia. [25] Similarly for shoulder arthroscopic surgeries, adding an interscalene block, superior trunk block, or a high thoracic erector spinae plane block could provide opioid-sparing analgesia and also $[26,27]$ many published research articles have investigated the efficacy of fascial plane blocks like transverse abdominis plane (TAP) block, rectus sheath block, quadratus lumborum block (QLB), paravertebral block (PVB) as a component of the ERAS pathway. The results reveal that not only the blocks are safe and effective, but they also are opioid-sparing and facilitate early recovery and mobilization. [2-33] Studies have demonstrated that a continuous TAP infusion is non-inferior to continuous TEA in patients undergoing colorectal surgeries. Single-shot TAP block was found to provide improved postoperative analgesia after LSCS which was although lesser compared to ITM, but had lesser side effects when compared to ITM. $[34,35]$ Fields et al used liposomal bupivacaine for TAP blocks in patients undergoing colorectal surgeries in the ERAS program and found that there was reduced opioid use and lesser hospital stay. [36] These blocks should be used as a component of the ERAS pathway based on the skills and resources available. These blocks could be single-shot or in the form of continuous infusion via indwelling catheters.

A lumbar erector spinae plane block (ESPB) is slowly gaining popularity as an analgesic modality for upper and lower abdominal surgeries like cholecystectomy, hiatus hernia repair. Although the block appears safe and effective, the available literature is in the form of case reports and series. To date, there is no well-designed RCT that has explored the premise in an ERAS pathway. $[37,38]$ ESPB at various levels have been successfully employed and provided opioid-sparing analgesia after spine surgeries also.
RA techniques like pectoral nerve blocks (PECS $1 / 2$ ), thoracic PVB, thoracic ESPB, serratus anterior plane block (SAPB) have been established as excellent, opioid-sparing interventions as a part of MMA for breast surgeries which have been attested by several RCTs and review articles. [4146] Not only do RA techniques provide opioid-sparing analgesia, reduced incidence of PONV, the propensity of post-mastectomy pain is also reduced if acute post-surgical pain is managed effectively. [47, 48]

To conclude, ERAS society strongly advocates the use of opioid-sparing MMA for any surgery and RA is an important component of MMA. Based on feasibility, expertise, and indication, some RA techniques should be offered to all the patients undergoing surgery to facilitate faster recovery, early ambulation, and discharge from the hospital. At present many centers are applying pathways laid by ERAS society in practice but still are defaulting in applying one important component i.e., documentation and audit. We suggest implementation, documentation, and continuous audit of all ERAS pathways for understanding the lacunae and thus improvise in the future. 


\section{Conflict of interest: Nil Source of support: None}

\section{References}

1. Ljungqvist O, Scott M, Fearon KC. Enhanced Recovery After Surgery: A Review.JAMA Surg. 2017; 152:292-8.

2.https://erassociety.org/guidelines/list-of-guidelines/Last accessed on 20th July,2021.

3. Joliat GR, Ljungqvist O, Wasylak T, Peters O, Demartines N. Beyond surgery: clinical and economic impact of Enhanced Recovery After Surgery programs. BMC Health Serv Res. 2018; 18:1008.

4. Mehdiratta L, Mishra SK, Vinayagam S, Nair A. Enhanced recovery after surgery (ERAS).... still a distant speck on the horizon! Indian J Anaesth. 2021; 65:93-6.

5. Ljungqvist O, Hubner M. Enhanced recovery after surgery-ERASprinciples, practice and feasibility in the elderly. Aging Clin Exp Res. $2018 ; 30: 249-252$.

6. Helander EM, Webb MP, Bias M, Whang EE, Kaye AD, Urman RD. Use of Regional Anesthesia Techniques: Analysis of Institutional Enhanced Recovery After Surgery Protocols for Colorectal Surgery. J Laparoendosc Adv Surg Tech A. 2017; 27:898-902.

7. Thapa P, Euasobhon P. Chronic postsurgical pain: current evidence for prevention and management. Korean J Pain. 2018;31:155-73.

8. Correll D. Chronic postoperative pain: recent findings in understanding and management. F1000Res. 2017; 6:1054.

9. Feldheiser A, Aziz O, Baldini G, Cox BP, Fearon KC, Feldman LS et al. Enhanced Recovery After Surgery (ERAS) for gastrointestinal surgery, part 2: consensus statement for anaesthesia practice. Acta Anaesthesiol Scand. 2016; 60:289-334.

10. Kaye AD, Urman RD, Rappaport Y, Siddaiah H, Cornett EM, Belani K et al. Multimodal analgesia as an essential part of enhanced recovery protocols in the ambulatory settings. J Anaesthesiol Clin Pharmacol. 2019;35(Suppl 1):S40-S45.

11. Simpson JC, Bao X, Agarwala A. Pain Management in Enhanced Recovery after Surgery (ERAS) Protocols. Clin Colon Rectal Surg. 2019; 32:121-8.

12. Tan M, Law LS, Gan TJ. Optimizing pain management to facilitate Enhanced Recovery After Surgery pathways. Can J Anaesth. 2015; 62:203-18.

13. Beverly A, Kaye AD, Ljungqvist O, Urman RD. Essential Elements of Multimodal Analgesia in Enhanced Recovery After Surgery (ERAS) Guidelines. AnesthesiolClin. 2017;35: e115-e143.

14. Dunkman WJ, Manning MW. Enhanced Recovery After Surgery and Multimodal Strategies for Analgesia. Surg Clin North Am. 2018; 98:117184.

15. Al-Mazrou AM, Kiely JM, Kiran RP. Epidural analgesia in the era of enhanced recovery: time to rethink its use? Surg Endosc. 2019; 33:21972205.

16. Borzellino G, Francis NK, Chapuis O, Krastinova E, Dyevre V, Genna M. Role of Epidural Analgesia within an ERAS Program after
Laparoscopic Colorectal Surgery: A Review and Meta-Analysis of Randomised Controlled Studies. Surg Res Pract. 2016; 2016:7543684.

17. Rosen DR, Wolfe RC, Damle A, et al. Thoracic Epidural Analgesia: Does It Enhance Recovery? Dis Colon Rectum. 2018; 61:1403-9.

18. Koning MV, Teunissen AJW, van der Harst E, Ruijgrok EJ, Stolker RJ. Intrathecal Morphine for Laparoscopic Segmental Colonic Resection as Part of an Enhanced Recovery Protocol: A Randomized Controlled Trial. Reg Anesth Pain Med. 2018; 43:166-73.

19. Kjølhede P, Bergdahl O, Borendal Wodlin N, Nilsson L. Effect of intrathecal morphine and epidural analgesia on postoperative recovery after abdominal surgery for gynecologic malignancy: an open-label randomised trial. BMJ Open. 2019;9: e024484.

20. Tang JZJ, Weinberg L. A Literature Review of Intrathecal Morphine Analgesia in Patients Undergoing Major Open Hepato-Pancreatic-Biliary (HPB) Surgery. Anesth Pain Med. 2019;9: e94441.

21. Wainwright TW, Gill M, McDonald DA, et al. Consensus statement for perioperative care in total hip replacement and total knee replacement surgery: Enhanced Recovery After Surgery (ERAS ${ }^{\circledast}$ ) Society recommendations. Acta Orthop. 2020; 91:3-19.

22. Blocks such as iPACK and adductor canal block are preferred over femoral and sciatic nerve blocks after TKA as they do not interfere with early ambulation which is desirable after TKA.

23. Kumar L, Kumar AH, Grant SA, Gadsden J. Updates in Enhanced Recovery Pathways for Total Knee Arthroplasty. Anesthesiol Clin. 2018; 36:375-86.

24. Oseka L, Pecka S. Anesthetic Management in Early Recovery After Surgery Protocols for Total Knee and Total Hip Arthroplasty. AANA J. 2018; 86:32-9.

25. Edwards MD, Bethea JP, Hunnicutt JL, Slone HS, Woolf SK. Effect of Adductor Canal Block Versus Femoral Nerve Block on Quadriceps Strength, Function, and Postoperative Pain After Anterior Cruciate Ligament Reconstruction: A Systematic Review of Level 1 Studies. Am J Sports Med. 2020; 48:2305-13.

26. Hewson DW, Oldman M, Bedforth NM. Regional anaesthesia for shoulder surgery. BJA Educ. 2019; 19:98-104.

27. Diwan S, Nair A. Erector Spinae Plane Block for Proximal Shoulder Surgery: A Phrenic Nerve Sparing Block!. Turk J Anaesthesiol Reanim. 2020; 48:331-3.

28. Kim AJ, Yong RJ, Urman RD. The Role of Transversus Abdominis Plane Blocks in Enhanced Recovery After Surgery Pathways for Open and Laparoscopic Colorectal Surgery.J Laparoendosc Adv Surg Tech A. 2017; 27:909-14.

29. Akerman M, Pejčić N, Veličković I. A Review of the Quadratus Lumborum Block and ERAS. Front Med (Lausanne). 2018; 5:44.

30. Guffey R, Keane G, Ha AY, et al. Enhanced Recovery With Paravertebral and Transversus Abdominis Plane Blocks in Microvascular Breast Reconstruction. Breast Cancer (Auckl). 2020; 


\section{$14: 1178223420967365$.}

31. Rotstein D, Park C, Khaitov S, Dickstein E. Rectus sheath catheters-a novel approach to perioperative analgesia for colorectal surgery in an enhanced recovery after surgery (ERAS) protocol: a case series. Int J Colorectal Dis. 2019; 34:1345-8.

32. Bakshi S, Mapari A, Paliwal R. Ultrasound-guided rectus sheath catheters: A feasible and effective, opioid-sparing, post-operative pain management technique: A case series. Indian J Anaesth. 2015; 59:118-20.

33. El-Boghdadly K, Madjdpour C, Chin KJ. Thoracic paravertebral blocks in abdominal surgery - a systematic review of randomized controlled trials. Br J Anaesth. 2016; 117:297-308.

34. Niraj G, Kelkar A, Hart E, Horst C, Malik D, Yeow C, Singh B, Chaudhri S. Comparison of analgesic efficacy of four-quadrant transversus abdominis plane (TAP) block and continuous posterior TAP analgesia with epidural analgesia in patients undergoing laparoscopic colorectal surgery: an open-label, randomised, non-inferiority trial. Anaesthesia.2014;

35. Mishriky BM, George RB, Habib AS. Transversus abdominis plane block for analgesia after Cesarean delivery: a systematic review and metaanalysis. Can J Anaesth. 2012; 59:766-78.

36. Fields AC, Weiner SG, Maldonado LJ, Cavallaro PM, Melnitchouk N, Goldberg J et al. Implementation of liposomal bupivacaine transversus abdominis plane blocks into the colorectal enhanced recovery after surgery protocol: a natural experiment. Int J Colorectal Dis. 2020; 35:1338.

37. Marija T, Aleksandar D. Erector spinae plane block in various abdominal surgeries: A case series. SaudiJ Anaesth. 2020; 14:528-30.

38. Hannig KE, Jessen C, Soni UK, Børglum J, Bendtsen TF. Erector Spinae Plane Block for Elective Laparoscopic Cholecystectomy in the Ambulatory Surgical Setting. Case Rep Anesthesiol. 2018; 2018:5492527.

39. Park S, ParkJ, Choi JW, et al. The efficacy of ultrasound-guided erector spinae plane block after mastectomy and immediate breast reconstruction with a tissue expander: a randomized clinical trial. Korean J Pain. 2021; 34:106-13.
40. ElHawary H, Joshi GP, Janis JE. Practical Review of Abdominal and Breast Regional Analgesia for Plastic Surgeons: Evidence and Techniques. Plast Reconstr Surg Glob Open. 2020;8: e3224.

41. Zhang Y, Liu T, Zhou Y, Yu Y, Chen G. Analgesic efficacy and safety of erector spinae plane block in breast cancer surgery: a systematic review and meta-analysis. BMC Anesthesiol.2021;21:59.

42. Chiu C, Aleshi P, Esserman LJ, et al. Improved analgesia and reduced post-operative nausea and vomiting after implementation of an enhanced recovery after surgery (ERAS) pathway for total mastectomy. BMC Anesthesiol. 2018; 18:41.

43. Parikh RP, Myckatyn TM. Paravertebral blocks and enhanced recovery after surgery protocols in breast reconstructive surgery: patient selection and perspectives.J Pain Res. 2018; 11:1567-81.

44. Afonso AM, Newman MI, Seeley N, et al. Multimodal Analgesia in Breast Surgical Procedures: Technical and Pharmacological Considerations for Liposomal Bupivacaine Use. Plast Reconstr Surg Glob Open. 2017;5: e1480.

45. Diwan S, Koh WU, Chin KJ, Nair A. Bilateral high thoracic continuous erector spinae plane blocks for postoperative analgesia in a posterior cervical fusion. SaudiJ Anaesth. 2020; 14:535-7.

46. Qiu Y, Zhang TJ, Hua Z. Erector Spinae Plane Block for Lumbar Spinal Surgery: A Systematic Review.J Pain Res. 2020; 13:1611-9.

47. Chiu C, Aleshi P, Esserman LJ, et al. Improved analgesia and reduced post-operative nausea and vomiting after implementation of an enhanced recovery after surgery (ERAS) pathway for total mastectomy. BMC Anesthesiol. 2018; 18:41.

48. Garg R. Regional anaesthesia in breast cancer: Benefits beyond pain. Indian J Anaesth. 2017; 61:369-72. 